\title{
Nonlinear noncoercive equations and applications
}

\section{P. DRÁBEK}

Es werden periodische Lösungen der nichtlinearen Balkengleichung

$$
\beta u_{t}+u_{t t}+u_{x x x x}-\lambda u+\varphi(u) \stackrel{!}{=} f
$$

in Abhängigkeit von einer nichtlinearen Funktion $\varphi: \mathbf{R} \rightarrow \mathbf{R}$ betrachtet. Die Untersuchungen schließen sich ān eine Arbeit von Fočfk [6] an und enthalten gegenüber dieser einigè neue Ergebnisse.

Рассматриваются периодические решения нелиненінго уравиения балки

$$
\beta u_{t}+u_{t t}+u_{x \times x x}-\lambda \dot{u}+\varphi(u)=f
$$

в аависимости от нелинейной функции $\varphi: \mathbf{R} \rightarrow \mathbf{R}$. Исследования примыкают к работе S. Fočtz [6] и включают в себя некоторые новые результаты, не содержациеся в этой -работе.

This paper deals with the periodic solvability of the nonlinear beam equation

$$
\beta u_{t}+u_{t l}+u_{x \times x x}-\lambda u+\varphi(u)=f,
$$

which depends on non-linear $\varphi: \mathbf{R} \rightarrow \mathbf{R}$. This paper continues the subject of the paper by S. Foč́́k [6]. We present some new methods and results which are not included in [6].

\section{1.' Introduction}

This paper continues the subject of the paper by S. Fočin [6]. We shall study, as in [6], problems which have their abstract formulation as an equation:

$$
T u=f,
$$

where $\dot{T}$ is operator acting from a Banach space $X$ into a Banach space $Z, T$ being of the form

$$
T u \stackrel{\prime}{=} L u+S u
$$

where $L$ is linear and $S$ is nonlinear. We are interested in the case when $T$ does not satisfy the coercivity condition

$$
\lim _{\|u\|_{X} \rightarrow+\infty}\|T u\|_{z}=+\infty .
$$

Typical examples of the operator equation (1.1) with condition (1.2) are the following problems. Let $\lambda$ be a real number, $\omega>0, \beta>0$, and let $\psi$, be a real valued continuous function. 
Boundary value problems for ordinary differential equations:

$$
\begin{aligned}
& -u^{\prime \prime}(x)-\lambda u(x)+\varphi(u(x))=f(x), \quad x \in(0, \pi) \\
& u(0)=u(\pi)=0 .
\end{aligned}
$$

Periodic problems for ordinary differential equations:

$$
\left.\begin{array}{l}
-u^{\prime \prime}(x)-i u(x)+\varphi(u(x))=f(x), \quad x \in(0, \pi) \\
u(0)=u(\pi), u^{\prime}(0)=u^{\prime}(\pi) .
\end{array}\right\}
$$

Boundary value problems for partial differential equations of elliptic type:

$$
\left.\begin{array}{l}
-\Delta u(x)-i u(x)+\dot{\varphi}(u(x))=f(x), \quad x \in \Omega \\
\dot{u}(x)=0, \quad x \in \partial \Omega,
\end{array}\right\}
$$

where $\Omega_{j}$ is a sufficiently smooth bounded domain in $N$-dimensional space.

One can consider higher order equations of the type (1.4)-(1.6) and also another type of boundary conditions than Dirichlet ones in (1.6).

Periodic solutions of the boundary value problem for the nonlinear heat equation:

$$
\begin{aligned}
& u_{t}(t, x)-u_{x \grave{x}}(t, x)-\lambda u(t, x)+\varphi(\ddot{u}(t, x)) . \\
& =f(t, x), \quad(t, x) \in Q:=(-\infty,+\infty) \times(0, \pi) \\
& \dot{u}(t, 0)=u(t, \pi)=0, \quad t \in(-\infty,+\infty), \\
& u(t+\omega, x)=u(t, x), \quad(t, x) \in Q .
\end{aligned}
$$

-Periodic solutions of the nonlinear telegraph equation:

$$
\left.\begin{array}{l}
\beta u_{t}(t, x) \pm u_{t t}(t, x)-u_{x x}(t, x)-\lambda u(t, x)+\varphi(u(t, x)) \\
=t(t, x), \cdot t, x \in(-\infty,+\infty), \\
u(t, x)=u(t+2 \pi, x)=u(t, x+2 \pi), \quad t, x \in(-\infty,+\infty) .
\end{array}\right\}
$$

Periodic solutions of the nonlinear beam, equation:

$$
\left.\begin{array}{l}
\beta u_{t}(t, x)+u_{t t}(t ; x)+u_{x x x x}(t, x)-\lambda u(t, x)+\varphi(u(t, x))=f(t, x), \\
u(t+2 \pi, x+2 \pi)=u(t+2 \pi, x)=u(t, x+2 \pi) \\
=u(t, x)(t, x \in(-\infty,+\infty)) .
\end{array}\right\}
$$

In the previous examples, the nonlinear operator $S$ is given by the nonlinear part $\varphi(u)$ of the problem considered and the operator $L$ is defined by the linear part, i.e. it is given by

$$
\begin{aligned}
& u \mapsto-u^{\prime \prime}-\lambda u \text { in (1.4) and (1.5), } \\
& u \mapsto-\Delta u-\lambda u \text { in (1.6), } \\
& u \mapsto u_{t}-u_{x x}-\lambda u \text { in (1.7), } \\
& u \mapsto \beta u_{t}+u_{t}-u_{x x}-\lambda u \text { in (1.8), } \\
& u \mapsto \beta u_{i}+u_{t t}+u_{x x x x}-\lambda u \text { in (1.9). }
\end{aligned}
$$

We present some methods and results (which are not included in [6]) about the solvability of the previous types of nonlinear equations. As in [6] we choose (1.9) as the model for the explanation of these methods. The methods used-here for solving (1.9) can be applied also for (1.4)-(1.8). The reason for choosing (1.9) is the same as in [6]. 


\section{Preliminaries}

In the sequel we shall denote by I the open interval $(0,2 \pi)$. Further, $\mathbf{N}, \mathbf{Z}$ and $\mathbf{R}$ will denote the set of positive integers, integers and real numbers, respectively.'Put

$$
\mathbf{I}^{2}=\mathbf{I} \times \mathbf{I}
$$

and analogously for other sets.

Before starting a precise definition of a periodic solution of the nonlinear beam equation

$$
\beta u_{t}+u_{t t}+u_{x x x x}+\varphi(u)=h(t, x),
$$

we introduce, in the same way as in [6], the suitable function spaces.

Denote, by $\mathbf{H}$ the space of all measurable real valued functions $u(t, x)$ defined alpiost everywhere on $\mathbf{R}^{2}$ which are $2 \pi$-periodic in the variables $t$ and $x$, i.e.

$$
u(t+2 \pi, x+2 \pi)=u(t+2 \pi, x)=u(t, \dot{x}+2 \pi)=u(t, x)
$$

for almost all $(\ell, x) \in \mathbf{R}^{2}$; and which are square integrable over $\mathbf{I}^{2}$. Introducing the inner product

$$
\langle h, k\rangle=\int_{\mathbf{I}^{2}} h(t, x) k(t, x) d t d x \quad(h, k \in \mathbf{H}),
$$

$\mathbf{H}$ becomes a Hilbert space. Its norm we denote by $\|\cdot\|_{\boldsymbol{H}}$, i.e.

$$
\|h\|_{\mathbf{H}} \doteq\langle h, h\rangle^{1 / 2}, \quad h \in \mathbf{H} .
$$

Iet $\tilde{\mathbf{H}}=\mathbf{H}+i \mathbf{H}$ be the complexification of the space $\mathbf{H}$. It is easy to see that

$$
\left\{e^{i(m x+n t)}:(m, n) \in \mathbf{Z}^{2}\right\}
$$

forms a complete orthogonal system in $\tilde{\mathbf{H}}$. Thus arbitrary $h \in \mathbf{H}$ can be expressed by

$$
h(t, x)=\sum_{i m, n) \in \mathbf{Z}^{3}} h_{m, n} e^{i(m x+n t)}
$$

(the convergence is in the space $\tilde{\mathbf{H}}$ ), where

$$
\sum_{(m, n) \in \mathbf{Z}^{2}}\left|h_{m, n}\right|^{2}<\infty, \quad h_{m, n}=\overline{h_{-m,-n}} .
$$

Tet $p, r$ be nonnegative integers. By $C_{2 \pi}^{p, r}$ we mean the set of all continuous functions $u(t, x)$ defined on $\mathbf{h}^{2}$ which are $2 \pi$-periodic in both variables, and sich that the partial derivatives by $t$ up to the order $p$. and the partial derivatives by $x$ up to the order $r$ are continuous on $\mathbf{R}^{2}$. With the norm

$$
\|u\|_{\mathbf{C}_{2 \pi}^{0.0}}^{0.0}=\max _{(t, x) \in \mathbf{R}^{2}}|u(t, x)|
$$

- $\mathbf{C}_{2 .}^{0.0}$ beconies a Banach space.

Definition 2.1: Let $p, r \in \mathbf{N} \cup\{0\}$. Define

$$
\mathbf{H}^{p, \tau}=\left\{h \in \mathbf{H}: \sum_{(m, n) \in Z^{2}}\left(m^{2 r}+n^{2 p}\right)\left|h_{m, n}\right|^{2}<\infty\right\} .
$$

$\mathbf{H}^{p, r}$ with the norm

$$
\|h\|_{\mathbf{H}, r}=\left(\sum_{(m, n) \in \mathbf{Z}^{2}}\left(m^{2 r}+n^{2 p}\right)\left|h_{m, n}\right|^{2}\right)^{1 / 2}
$$

is a Banach space which is nothing other than a Sobolev space of periodic functions (see [10]). 
Definition 2.2 (Generalized periodic solutions): Let $\varphi$ be a continuous function defined on $\mathbf{R}$. Suppose that there exist $\alpha_{1}, \alpha_{2} \geqq 0$ such that

$$
|\varphi(z)| \leqq \alpha_{1}+\alpha_{2}|z|, \quad z \in \mathbf{R} .
$$

Let $\beta>0$ and $h \in \mathbf{H}$. A generalized periodic solution (GPS) of (2.1) is a real function ,$u \in \mathbf{H}$ such that for all $v \in \mathbf{C}_{2 \pi}^{2,4}$ one has

$$
\left\langle u,-\beta v_{t}+v_{u}+\dot{v}_{x x x x}\right\rangle=\langle h-\varphi(u), v\rangle .
$$

Remark 2.1 : (i) The growth condition (2.2) is necessary for a Némytskij's operator

$$
u \mapsto \varphi(u)
$$

acting from $H$ into $H$ (see e.g. [6, 7]).

(ii) Using integration by parts in (2.3) we can prove that if $u \in \mathbf{C}_{2 \pi}^{2,4}$ is a GPS of (2:1), then the equation (2.1) is fulfilled on $R^{2}$ (i.e. $u$ is "a classical solution" of (2.1)): On the other hand, an arbitrary GPS $u \in \mathbf{H}$ has better properties '(see [6: Th. 2.4, 2.5]).

Let us denote

$$
\sigma=\left\{q \in \mathbf{N} \cup\{0\}: q^{1 / 4} \in \mathbf{N}\right\}
$$

The following two theorems are proved in [6]:

Theorem 2.1: Let $\lambda \in$ R. Then the equation

$$
\beta u_{t}+u_{t t}+u_{x x x x}-\lambda u=h
$$

has for arbitrary $h \in \mathbf{H}$ a unique GPS $u \in \mathbf{H}$ if and only if $\lambda \notin \sigma$;

Theorem 2.2: Let $\lambda=q \in \sigma$. Denote by $\mathscr{H}_{q}$ and $\mathscr{H}_{q}{ }^{\perp}$ two closed orthogonal subspaces of $\mathbf{H}$ with the following properties:

$$
\begin{aligned}
& \mathscr{H}_{q}=\left\{h \in \mathbf{H}: h_{0 . q}=h_{0,-q}=0\right\} \\
& \mathscr{H}_{q}{ }^{1}=\text { linear hull of }\left\{\sin q^{1 / 4} x, \cos q^{1 / 4} x\right\} \text { provided } q \neq 0 ; \\
& \mathscr{H}_{0}{ }^{\perp}=\text { linear } \text { hull of constant functions. }
\end{aligned}
$$

Then for an arbitrary $h \in \mathscr{H}_{q}$ there exists a unique GPS $u \in \mathscr{H}_{q}$ of (2.4).

Put $\mathbf{C}_{q}=\mathbf{C}_{2 \pi}^{0,0} \cap \mathscr{H}_{q}$ and define the mapping

$$
\tilde{T}_{q}: \mathscr{H}_{q} \rightarrow \mathscr{H}_{q}, \tilde{T}_{q}: h \mapsto u,
$$

where $u$ is the unique G.PS of (2.4). Then

(i) $\tilde{T}_{q}$ is linear, $\operatorname{Im} \tilde{T}_{q} \subset \mathbf{C}_{q}$;

(ii) The mappings $\tilde{T}_{q}: \mathscr{H}_{q} \rightarrow \mathscr{H}_{q}, \tilde{T}_{q}: \mathscr{H}_{q} \rightarrow \mathbf{C}_{q}, \tilde{T}_{q} \mid \mathbf{c}_{q}: \dot{\mathbf{C}}_{q} \rightarrow \mathbf{C}_{q}$, are completely continuous (where the norm $\|\cdot\| \boldsymbol{c}_{2 \pi}^{0.0}$ is introduced in the space $\mathbf{C}_{q}$ and the norm. $\|\cdot\|_{\mathbf{H}}$ in $\left.\mathscr{H}_{q}\right)$.

(iii) If $p, r \in \mathbf{N} \cup\{0\}$ then

$$
\tilde{T}_{q}\left(\mathbf{H}^{p, q} \cap \mathscr{H}_{q}\right) \subset\left(\mathbf{H}^{p+1, r+1} \cap \mathscr{H}_{q}\right) .
$$




\section{Bounded nonlinearities}

Assumptions: We prove the existence and multiplicity of GPSs of

$$
\beta u_{t}+u_{u t}+u_{x x x x}+\varphi(u)=h,
$$

where $\beta>0, h \in \mathbf{H}$ and $\varphi: \mathbf{R} \rightarrow \mathbf{K}$ is a continuous function with finite limits

$$
\varphi( \pm \infty)=\lim _{z \rightarrow \pm \infty} \varphi(z)
$$

Moreover let us suppose

$$
\varphi(z) z \geqq 0, \quad \varphi(-\infty) \leqq 0 \leqq \varphi(+\infty)
$$

(the case $\varphi(z) z \leqq 0, \varphi(+\infty) \leqq 0 \leqq \varphi(-\infty)$ can be treated similarly). Suppose there exists $\delta>0$ such that

$$
\begin{array}{ll}
\varphi(z) \geqq \varphi(+\infty), & z \geqq \delta, \\
\varphi(z) \leqq \varphi(-\infty), & z \leqq-\delta .
\end{array}
$$

A typical example of such a function $\varphi$ is

$$
\dot{\varphi}(z)=z e^{-z^{2}}, \quad z \in \mathbf{R} .
$$

In contrast to [6]; we make no assumptions about the limits

$$
\lim _{z \rightarrow \pm \infty}(\varphi(z) \div \varphi( \pm \infty)) z
$$

Remark 3.1: Denote by $\mathbf{P}_{0}$ the orthogonal projection from $\mathbf{H}$ onto $\mathscr{H}_{0}{ }^{\perp}$. Put

$$
P_{0}^{c}: u \mapsto u-\mathbf{P}_{0} u, \quad u \in \mathbf{H} .
$$

The mapping $P_{0} c$ is the orthogonal projection from $\mathbf{H}$ onto $\mathscr{H}_{0}$. Then for each $h \in \mathbf{H}$ there exists an $s \in \mathbf{R}$ and an $h_{1} \in \mathscr{H}_{0}$ such that

$$
\begin{array}{r}
\quad h=s+h_{1}, \\
s=P_{0} h, h_{1}{ }^{\prime}=P_{0} c h .
\end{array}
$$

Theorem 3.1: For each $h_{1} \in \mathscr{H}_{0}$ there exist real numbers $T_{1} \leqq 0 \leqq T_{2}$ such that.

(i) the equation (3.1) has at least one GPS for $h=s+h_{1}$ with $s \in\left(T_{1}, T_{2}\right)$ in the case $T_{1}<T_{2}$, moreover (3.1) has at least one GPS for $h=h_{1}$;

(ii) the equation (3.1) has at least two distinct GPSs for $h=s+h_{1}$. with $s . \in\left(T_{1}, \varphi(-\infty)\right) \cup\left(\varphi(+\infty), T_{2}\right)$ in the case $T_{1}<T_{2}$.

Proof: Put

$$
G: u \mapsto \varphi(u), \quad u \in \mathbf{H} .
$$

Then it is easy to see that the equation (3.1) is solvable (in the sense of Definition 2.2) if and only if the following bifurcation system is solvable:

$$
\left.\begin{array}{l}
v+\tilde{T}_{0} P_{0} c G(w+v)^{-}=\tilde{T}_{0} P_{0} c h ; \\
P_{0} G(w+v)=P_{0} h,
\end{array}\right\}
$$

where $\dot{w}=P_{0} u, v=P_{0} c_{u} u \in \mathbf{H}$ (see e.g. [6]). 
Let us denote $w_{1}(t, x)=\frac{1}{4 \pi^{2}}$, for $(t, x) \in \mathbf{I}^{2}$. Then

$$
\int_{1} w_{1}(t, x) d t d x=1 \text {. }
$$

and for each $w \in \mathscr{H}_{0} \perp$ there exists a real number $\tau$ such that

$$
w=\tau w_{1} .
$$

Let $h_{1} \in \mathscr{H}_{0}$ be arbitrary but fixed. Because the function $\varphi$ is continuous and bounded on $\mathbf{R}$ then for a possible solution $v$ of the first equation in (3.2),

$$
\|v\|_{\mathbf{c}_{2 \pi}^{0,0}} \leqq c
$$

(see Theorem 2.2 (ii)), where the constant $c>0$ is independent of $v$. Let us consider a ball $\mathbf{B}_{R}(0)$ with its centre at the origin and with sufficiently large radius $R>0$. Then for each $w \in \mathscr{H}_{0}{ }^{\perp}$, and $v \in \partial \mathbf{B}_{R}(0)$

$$
v+\tilde{T}_{0} P_{0} c G(w+v)-\tilde{T}_{0} P_{0} c h \neq 0
$$

By a standard application of the Leray-Schauder degree theory we can prove (see [5]) that for each $w \cdot \epsilon, \mathscr{H}_{0}{ }^{c}$ there is at least one $v \in \mathscr{H}_{0}$ satisfying the first equation in (3.2). Let us define

$$
\mathbf{S}=\left\{(\tau, v) \in \mathbb{R} \times \mathscr{H}_{0}: w=\tau w_{1} \text { and } v \text { satisfies the first equation in (3.2) }\right\} \text {. }
$$

Then the solutions of (3.1) are such $u=\tau w_{1}+v$ that $(\tau ; v) \in \mathbf{S}$ and

where

$$
\dot{\psi}(\tau, v)=\left\langle h, w_{1}\right\rangle
$$

$$
\psi \quad \psi(\tau, v)^{\prime}=\int_{I^{2}} p\left(\tau w_{1}+v\right) w_{1}
$$

is a real continuous function defined on $\mathbf{S}$. For fixed $\tau \in \mathbf{R}$ put ,

$$
\begin{aligned}
& \underline{\tau}(\tau)=\inf _{(\tau, v) \in \mathbf{S}} \psi(\tau, v) \quad \text { and } \quad \bar{\tau}(\tau)=\sup _{(\tau, v) \in \mathbb{S}} \psi(\tau, v) ; \\
& T_{1}^{\prime}=\inf _{\boldsymbol{\tau} \in \mathbf{R}} \bar{\tau}(\tau) \quad \text { and } \quad T_{\mathbf{2}}=\sup _{\boldsymbol{\tau} \in \mathbf{R}} \underline{\tau}(\tau) .
\end{aligned}
$$

Let us remark. that if for some $v \in \mathscr{H}_{0}$ there exists $\tau \in \mathbf{R}$ such that $(\tau, v) \in \mathbf{S}$ then

$$
\|v\|_{\mathbf{c}_{2 \pi}^{0,0}} \leqq c
$$

(see (3.3)). So the assumptions at the beginning of this section guarantee the inequality $T_{1} \leqq 0 \leqq T_{2}$ :

Suppose, now, that $\dot{T}_{1}<T_{2}$ and $s \in\left(T_{1}, T_{2}\right)$. Then according to (3.4) there exist $\tau_{1}, \tau_{2} \in \mathbf{R}$ such that

$$
\psi\left(\tau_{1}, v\right)>s \quad \text { and } \quad \psi\left(\tau_{2}, v\right)<s
$$

for all $\left(\tau_{1}, v\right) \in \dot{S},\left(\tau_{2}, v\right) \in \mathbf{S}$. To prove that the equation (3.1) has at least one GPS for $h=s+h_{1}$, we need the following lemma.

I.emma 3.1: For each real number $\bar{\beta}>0$ there exists a connected subset $\mathbf{S}_{\bar{\beta}} \subset \mathbf{S}$ such that proj $_{\mathbf{R}} \mathbf{S}_{\bar{\beta}} \supset[-\bar{\beta}, \bar{\beta}]$ (where proj $\mathbf{j}_{\mathbf{R}} \mathbf{S}_{\bar{\beta}}$ denotes the projection of the set, $\mathbf{S}_{\bar{\beta}}$ onto $\mathbf{R}$ ).

For the proof of Lemma 3.1 see $[1,4]$. 
Having the assertion of Lemma 3.1 , we can choose $\bar{\beta}>0$ such that

$$
\text { . } \bar{\beta}>\max \left\{\left|\tau_{1}\right|,\left|\tau_{2}\right|\right\} \text {. }
$$

The fact that $\psi$ is continuous on the connected subset $\mathbf{S}_{\bar{\beta}}$ inplies the existence of at least one pair $(\tau, v) \in \mathrm{S}_{\bar{\beta}}$ such that

$$
\psi(\tau, v)=s .
$$

Then $u=\tau w_{1}+v$ is the desired solution of (3.1). According to the assumptions on $\varphi$ there exists $\tau_{0} \in \mathbf{R}$ such that

$$
\psi\left(\tau_{0}, v\right) \leqq 0 \quad \text { and } \quad \psi\left(-\tau_{0}, v\right) \geqq 0,
$$

for all $\left(\tau_{0}, v\right) \in \mathbf{S},\left(-\tau_{0}, v\right) \in \mathbf{S}$. Using Lemma 3.1 we prove the existence of at least one GPS of (3.1) with $s=0$ and the assertion (i) is proved.

We shall prove, now, the assertion (ii). Let $T_{1}<T_{2}$ and $s \in\left(T_{1}, \varphi(-\infty)\right)$. Then according to (3.4) there exists $\tau_{3} \in \mathbf{R}$ such that $\varphi\left(\tau_{3}, v\right)<s$ for all $\left(\tau_{3}, v\right) \in \mathbf{S}$. It is , sufficient to prove the existence of $\tau_{4}, \tau_{5} \in \mathbf{R}$ such that $\psi\left(\tau_{i}, v\right)>s$ for all $\left(\tau_{i}, v\right) \in \mathbf{S}$, $i=4,5$. Then.using Lemma 3.1 we obtain at least two distinct solutions of (3.1). Put

$$
\begin{aligned}
& \mathbf{I}_{n, x}^{2}=\left\{(t, x) \in \mathbf{I}^{2}: \tau w_{1}(t, x)+v(t, x) \leqq u \text { for all }(\tau, v) \in \mathbf{S}\right\}, \\
& \mathbf{I}_{-n, \tau}^{2}=\left\{(t, x) \in \mathbf{I}^{2}: \tau w_{1}(t, x)+v(t, x) \geqq-n \text { for all }(\tau, v) \in \mathbf{S}\right\} .
\end{aligned}
$$

It is easy to see that

$$
\lim _{t \rightarrow \pm \infty} \text { meas } \mathbf{I}^{2}{ }_{ \pm n, t}=0 \text { for each } n \in \mathbf{N}^{-}
$$

(see (3.3)). According to the assumptions on $\varphi$ we can choose for arbitrary $\varepsilon>0$ such $\tau_{0} \in \mathbf{R}$ and $n \in \mathbf{N}$ that for $\tau_{\mathbf{4}}=-\tau_{0}$

$$
\begin{aligned}
& \left|\int_{\mathbf{I}^{2} \backslash \mathbf{I}_{-\pi, \tau_{1}}^{2}} \varphi\left(\tau_{4} w_{1}+v\right) w_{1}-\varphi(-\infty)\right|<\frac{\varepsilon}{2}, \\
& \left|\int_{\mathbf{I}_{-\pi, r_{1}}^{2}} \varphi\left(\tau_{4} w_{1}+v\right) w_{1}\right|<\frac{\varepsilon}{2},
\end{aligned}
$$

and for $\tau_{5}=\tau_{0}$

$$
\begin{aligned}
& \left|\int_{\mid \mathbf{I}^{1} \mathbf{I}_{n_{0}, z_{5}}^{2}} \varphi\left(\tau_{5} w_{1}+v\right) w_{1}-\varphi(+\infty)\right|<\frac{\varepsilon}{2}, \\
& \left|\int_{\mathbf{I}_{n_{1}, \tau_{5}}^{2}} \varphi\left(\tau_{5} w_{1}+v\right) w_{1}\right|<\frac{\varepsilon}{2},
\end{aligned}
$$

for all $\left(\tau_{i}, v\right) \in \mathbf{S}, i=4,5$. From (3.5), (3.6) we obtain

$$
\left|\int_{1} \varphi\left(\tau_{4} w_{1}+v\right) w_{1}-\varphi(-\infty)\right|<\varepsilon \text { and }\left|\int_{1} \varphi\left(\tau_{5} \bar{w}_{1}+v\right) v_{1}-\varphi(+\infty)\right|<\varepsilon^{i}
$$

for all $\left(\tau_{i}, v\right) \in \mathrm{S}, i=4,5$. Put $\varepsilon=\frac{\varphi(-\infty)-s}{2}$. Then the last two inequalities imply that the function $\psi$ has desired property, i.e. $\psi\left(\tau_{i}, v\right)>s$ for all $\left(\tau_{i}, v\right) \in \mathbf{S}$, $i=4,5$. If $s \in\left(\varphi(+\infty), T_{2}\right)$, the proof of the assertion (ii) is analogous. This completes the proof of Theoreni 3.1 
Remark 3.2: Let us consider the equation

$$
\beta u_{t}+u_{t t}+u_{x x x x}+u e^{-u^{2}}=h \quad\left(h=s+h_{1}\right) .
$$

Then from Theorem $3.1^{\prime}$ it follows (by an easy calculation) that for each $h_{1}-\epsilon \mathscr{H}_{0}$ there exist $T_{1}\left(h_{1}\right)<0<T_{2}\left(h_{1}\right)$ such that $\left(3.1^{\prime}\right)$ has at least one GPS if $s \in\left(T_{1}, T_{2}\right)$ and $\left(3.1^{\prime}\right)$ has at least two distinct GPSs if $s \in\left(T_{1}, 0\right) \cup\left(0, T_{2}\right)$.

Remark 3.3: The existence of the solution of the boundary value problem for second order elliptic partial differential equations with analogous nonlinearity $\varphi(u)$ is proved in [5]. Existence and multiplicity results of such problems are proved in [2].

\section{Superlinear nonlinearities}

In this section we shall consider the generalized periodic solvability of the equation

$$
\beta u_{t}+\dot{u}_{t t}+u_{x x x x}-\lambda u+\varphi(u)=h,
$$

where $\lambda>0, \ddot{\varphi}$ is a continuous real valued function which is bounded on the interval $(-\infty, 0]$ and

$$
\lim _{z \rightarrow+\infty} \frac{\varphi(z)}{z}=+\infty
$$

Adding suitable constants on both sides of the equation (4.1), we may assume without loss of generality that

$$
\varphi(z) \geqq 0 \text { for all } z \in \mathbf{R} \text {. }
$$

As an example we can present the function $\varphi(z)=e^{z}, z \in \mathbf{R}$. Since there are no restrictions on the growth of $\varphi$ in $+\infty$, we must slightly modify the definition of a GPS (see Definition 2.2).

Definition 4.1 : Let $\beta>0$ and $h \in \mathbf{H}$. A generalized periodic solution (GPS) of (4.1) is a real function $u \in \mathbf{H}_{1}$ such that for all $v \in \mathbf{C}_{2 \pi}^{2.4}$ we have

$$
\left\langle u,-\beta v_{t}+v_{t t}+v_{x x x \boldsymbol{x}}\right\rangle=\int_{\mathbf{I}^{2}} h v-\int_{\mathbf{I}^{2}} \varphi(u) v+\int_{\mathbf{I}^{2}} \lambda . u v
$$

where

$$
\mathbf{H} \supset \mathbf{H}_{1}:=\left\{\dot{u} \in \mathbf{H}: \int_{\mathbf{1}^{2}} \varphi(u)<+\infty\right\}
$$

The main assertion of this section is the following theorem.

Theorem 4.1: Let $h \in \mathbf{H}, h=s+h_{1}$. Then there exist real numbers $T_{1}^{\prime}\left(h_{1}\right) \leqq T_{2}\left(h_{1}\right)$ and a bounded set. $\mathbf{M}\left(h_{1}\right) \subset\left[T_{1}\left(h_{1}\right), T_{2}\left(h_{1}\right)\right], T_{2}\left(h_{1}\right) \in \mathbf{M}\left(h_{1}\right)$ such that

(i) the equation (4.1) has at least two distinct GPSs if $s>T_{2}\left(h_{1}\right)$;

(ii) the equation (4.1) has at least one GPS if $s \in \mathbf{M}\left(h_{1}\right)$;

(iii) the equation (4.1) has no GPS if $s<I_{1}\left(h_{1}\right)$.

Proof: Let $h_{1} \in \mathscr{H}_{0}$ be arbitrary but fixed. We shall prove, first, that for fixed $\tau \in \mathbf{R}$ there exists at least one $v(\tau) \in \mathscr{H}_{0}$ such that

$$
\left\langle v(\tau) ;-\beta v_{t}+v_{\imath t}+v_{x x x x}\right\rangle=\int_{\mathbf{I}^{2}} h_{1} v-\int_{\mathbf{I}^{2}} \varphi\left(\tau w_{1}+v(\tau)\right) v+\lambda \int_{\mathbf{I}^{2}} v(\tau) v
$$

holds for each $v \in \mathbf{C}_{2 \pi}^{2,4} \cap \mathscr{H}_{0}$.

We insert now two lemmata. 
I.emma 4.1: Let $\mathscr{I} \subset \mathbf{R}$ be a bounded interval. Then there exists a constant $r>0$ such that for $u \in \mathscr{H}_{\mathbf{0}} \cap \mathbf{C}_{2 \pi}^{2,4},\|u\|_{\mathbf{H}}>r$ and $\tau \in \mathscr{I}$

$$
u \neq \sigma \tilde{T}_{0}\left(h_{1}-P_{0} c \dot{G}\left(\tau u_{1}+u\right)+\lambda u\right),
$$

for all $\sigma \in[0,1]$.

Proof: We argue by contradiction. Suppose for all $n \in \dot{N}$ there exists $\tilde{u}_{n} \in \mathscr{H}_{0}$ $\cap \mathbf{C}_{2 .,}^{2,4},\left\|\tilde{u}_{n}\right\| \dot{H} \geqq n, \tau_{n} \in \mathscr{I}$ and $\sigma_{n} \in[0,1]$. such that

$$
\tilde{u}_{n}=\sigma_{n} \tilde{T}_{0}\left(h_{1}-\dot{P}_{0} c G\left(\tau_{n} w_{1}+\tilde{u}_{n}\right)+\lambda \tilde{u}_{n}\right) \text {. }
$$

Applying $\tilde{T}_{0}^{-1}$ to both sides of (4.5) and taking the inner product with $\tilde{u}_{n}$ we obtain

$$
-\left\langle\tilde{T}_{0}^{-1} \tilde{u}_{n}, \tilde{u}_{n}\right\rangle=\sigma_{n}\left[\left\langle h_{1}, \tilde{u}_{n}\right\rangle-\left\langle G\left(\tau_{n} w_{1},+\tilde{u}_{n}\right), \tilde{u}_{n}\right\rangle+\lambda\left\langle\dot{\bar{u}}_{n}, \tilde{u}_{n}\right\rangle\right] .
$$

Letting $u_{n}=\frac{\bar{u}_{n}}{\left\|\tilde{u}_{n}\right\|_{\mathbf{H}}}$ and dividing by $\left\|\tilde{u}_{n}\right\|_{\mathbf{H}}^{2}$ we get

$$
\begin{aligned}
& \left\langle u_{n},-\beta\left(u_{n}\right)_{\ell}+\left(u_{n}\right)_{i}+\left(u_{n}\right)_{x x x z}\right\rangle+\sigma_{n}\left\|\bar{u}_{n}\right\|_{\mathbf{H}^{-1}}\left\langle G\left(\tau_{n} \dot{w}_{1}+\tilde{u}_{n}\right), u_{n}\right\rangle-\sigma_{n} \lambda \\
& =\sigma_{n}\left\|\bar{u}_{n}\right\|_{\mathbf{H}^{-1}}\left\langle h_{1}, u_{n}\right\rangle .
\end{aligned}
$$

This means that $\left\langle u_{n},-\beta\left(u_{n}\right)_{t}\left(u_{n}\right)_{t}+\left(u_{n}\right)_{x x x x}\right\rangle$ is a real number and so $\left\langle u_{n},-\beta\left(u_{n}\right)_{t}\right.$ $\left.+\left(u_{n}\right)_{t}+\left(u_{n}\right)_{x x x x}\right\rangle \geqq$ const. $>0$ for each $n \in \mathbf{N}$. From (4.6) we obtain

$$
\begin{aligned}
& \left\langle u_{n},-\beta\left(u_{n}\right)_{t}+\left(u_{n}\right)_{t i}+\left(u_{n}\right)_{x x x z}\right\rangle \\
& +\sigma_{n}\left\|\tilde{u}_{n}\right\|_{\mathbf{H}^{-1}}\left\langle G\left(\tau_{n} w_{1}+\tilde{u}_{n}\right), u_{n}\right\rangle-\sigma_{n} \hat{\lambda} \leqq\left\|h_{1}\right\|_{\mathbf{H}}\left\|\tilde{u}_{n}\right\|_{\mathbf{H}}^{-\mathbf{1}} .
\end{aligned}
$$

Since $\left\langle G\left(\tau_{n} w_{1}+\tilde{u}_{n}\right), u_{n}\right\rangle$ is bounded below (we assume $\dot{\varphi} \geqq 0$ and $\varphi$ is bounded on $(-\infty, 0])$,

$$
\left\langle u_{n},-\beta\left(u_{n}\right)_{t}+\left(u_{n}\right)_{t \prime}+\left(u_{n}\right)_{x x x x}\right\rangle \leqq\left(\lambda+\dot{\prime}^{\prime} \dot{\prime}\right)
$$

for sufficiently large $n \in \mathbf{N}$. We prove that there exists an $\alpha \in(0,1)$ such that $\left\|u_{n}{ }^{+}\right\|_{\mathbf{H}} \geqq \alpha$ for sufficiently large $n$. Suppose on the contrary that there is a subsequence of $\left\{u_{n}\right\}_{n-1}^{\infty}$, which we shall also denote by $\left\{u_{n}\right\}_{n-1}^{\infty}$, such that $\left\|u_{n}^{+}\right\|_{\mathbf{H}} \rightarrow 0$. From (4.7) we obtain that $\left\{u_{n}\right\}_{n-1}^{\infty}$ is bounded in the space $\mathbf{H}^{2,1}$. Since $\mathbf{H}^{2,1} \bigcirc \bigcirc \mathbf{H}$ (compact imbedding) we can suppose, after possibly 'passing to a suitable subsequence, that $u_{n} \stackrel{H}{\longrightarrow} \dot{u_{0}}$, . $\left\|u_{0}\right\|_{\mathbf{H}}=1$ and $u_{0} \leqq 0$ a.e. in $\mathbf{I}^{2}$. But this is a contradiction to the fact $u_{0} \in \mathscr{H}_{0}$.

Note that there exists such a constant $\gamma>0$ that.

$$
-\varphi(z) \geqq \frac{\lambda}{\alpha^{2}} z-\gamma, \text { for all } z \in \mathbf{R} \text {. }
$$

Further note' that i

$$
\left\langle\mathbf{G}\left(\tau_{n} w_{1}+\tilde{u}_{n}\right), u_{n}\right\rangle, \geqq\left\langle G\left(\tau_{n} w_{1}+\tilde{u}_{n}\right), u_{n}{ }^{+}\right\rangle-c_{1},
$$

because $\varphi$ is bounded on $(-\infty, 0], w_{1} \in L^{\infty}\left(\mathbf{I}^{2}\right)$ and $\left\{\tau_{n}\right\}$ is bounded. From (4.6) and '(4.8) we obtain

$$
\begin{aligned}
\left\|h_{1}\right\|_{\mathbf{H}} \geqq \text { const. }\left\|\tilde{u}_{n}\right\|_{\mathbf{H}}+\sigma_{n}\left\langle G\left(\tau_{n} w_{1}+\tilde{u}_{n}\right), u_{n}{ }^{+}\right\rangle-\sigma_{n} c_{1}-\sigma_{n} \lambda\left\|\tilde{u}_{n}\right\|_{\mathbf{H}} \\
\qquad \text { const. }\left\|\tilde{u}_{n}\right\|_{\mathbf{H}}+\sigma_{n}\left[\frac{\lambda}{\alpha^{2}} \int_{\mathbf{I}}^{1}\left(\tau_{n} w_{1}+\left\|\tilde{u}_{n}\right\|_{\mathbf{H}} u_{n}\right) \dot{u}_{n}{ }^{+}-\gamma \int_{\mathbf{I}} u_{n}{ }^{+}\right]
\end{aligned}
$$




$$
\begin{aligned}
& \geqq \text { const. }\left\|\tilde{u}_{n}\right\|_{\mathbf{H}}+\sigma_{n} \frac{\lambda}{\alpha^{2}} \alpha^{2}\|\tilde{u}\|_{\mathbf{H}}-c_{2}-\sigma_{n} \lambda \| \underset{\tilde{u}_{n}}{\|_{\mathbf{H}}} \\
& \geqq \text { const. }\left\|\tilde{u}_{n}\right\|_{\mathbf{H}}=c_{2} .
\end{aligned}
$$

The final inequality implies the boundedness of $\left\|\tilde{u}_{n}\right\|_{\mathbf{H}}$, which is a contradiction. This - proves the Lemma

Lemma 4.2: Let $\mathscr{I} \subset \mathbf{R}$ be a bounded interval. Then there exists a constant $r>0$

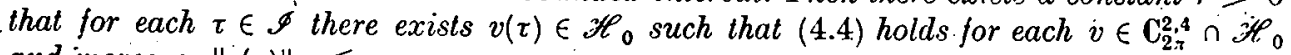
and moreover $\|\boldsymbol{v}(\tau)\|_{\mathbf{H}} \leqq r$.

Proof: Let $\tau \in \mathscr{I}$ be fixed. We use the Galerkin method to prove the existence of $v(\tau)$ : Choose $\mathrm{V}_{n} \subset \mathscr{H}_{0} \cap \mathbf{C}_{2,}^{\infty, \infty}$ such that $\operatorname{dim} \mathbf{V}_{n}=n, \mathbf{V}_{n} \subset \mathbf{V}_{n+1}$ and $\bigcup^{\infty} \mathbf{V}_{n}$ is dense in $\mathscr{H}_{0}$. A function $u_{n} \in V_{n}$ is called the Galerkin solution of (4.4) if $n=1$

$$
u_{n} \doteq \tilde{T}_{0}\left(h_{1}-P_{0} c G\left(\tau w_{1}+u_{n}\right)+\hat{\lambda} u_{n}\right) \text {. }
$$

From Lemma 4.1 and from the homotopy invariance property of the Brouwer degree, we obtain for each $n \in \mathbb{N}$ the existence of the Galerkin solution $u_{n}$ of (4.4) such that $\left\|u_{n}\right\|_{\mathbf{H}} \leqq r$. Then $\left\|h_{1}-P_{0}^{c} G\left(\tau w_{1}+u_{n}\right)+\lambda u_{n}\right\|_{\mathbf{H}} \leqq$ const. (we use the continuity of $\left.\widetilde{T}_{0}^{-1}\right)$. After possibly' passing to subsequences, we can suppose that

$$
h_{1}-\dot{P}_{0} c G\left(\tau w_{1}+u_{n}\right)+\lambda u_{n} \rightarrow u_{0} \in \mathscr{H}_{0} .
$$

According to the complete continuity of $\tilde{T}_{0}$. (Th. 2.2(ii)) and (4.9) we can suppose

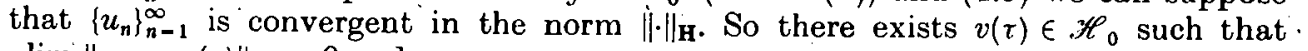
$\lim \left\|u_{n}-v(\tau)\right\|_{\mathbf{H}}=0$ and $n \rightarrow+\infty$;

$$
\|v(\tau)\|_{\mathbf{H}} \leqq r .
$$

It remains to prove that $\varphi\left(\tau w_{1}+\dot{v}(\tau)\right) \in L^{\mathbf{1}}\left(\mathbf{I}^{\mathbf{2}}\right)$ and

$$
\varphi\left(\tau w_{1}+u_{n}\right) \stackrel{L^{1}\left(\mathbf{I}^{2}\right)}{\rightarrow} \varphi\left(\tau\left(w_{1}+v(\tau)\right)\right. \text {. }
$$

Since $u_{n} \in \mathbf{V}_{n}$ is the Galerkin solution of (4.4) for each $n \in \mathbf{N}$, we obtain from (4.9)

$$
\int_{\mathbf{I}^{2}}\left|u_{n} \varphi\left(\tau w_{1}+u_{n}\right)\right| \leqq K\left\|u_{n}\right\|_{\mathbf{H}}{ }^{2}+\lambda\left\|u_{n}\right\|_{\mathbf{H}}+\left\|h_{\mathbf{1}}\right\|_{\mathbf{H}}\left\|u_{n}\right\|_{\mathbf{H}} \leqq \text { const. }
$$

(where $K$ is a constant independent of $n$ ).

We have proved that $u_{n} \stackrel{H}{\rightarrow} v(\tau)$ and so

$$
u_{n} \rightarrow v(\tau)^{-} \text {and } u_{n} \varphi\left(\tau w_{1}+u_{n}\right) \rightarrow v(\tau) \varphi\left(\tau w_{1}+v(\tau)\right) \text { a.e. in } \mathbf{I}^{2} \text {. }
$$

Having (4.10) Fatou's lemma implies that

$$
v(\tau) \varphi\left(\tau w_{1}+v(\tau)\right) \in L^{1}\left(\mathbf{I}^{2}\right) \text {. , }
$$

Then for each $k \in \mathbf{N}$ and $\varepsilon>0$ there exists such a $\delta>0$ that for each $\dot{\Omega} \subset \mathbf{I}^{2}$, meas $\Omega<\delta$ we obtain

$$
\int_{\Omega \cap\left(\left|u_{n}\right| \leqq k\right\}}\left|\varphi\left(\tau w_{1}+u_{n}\right)\right|<\frac{\varepsilon}{2}, \quad \frac{1}{k} \int_{\Omega \cap\left\{\left|u_{n}\right|>k\right\}}\left|u_{n} \varphi\left(\tau w_{1}+u_{n}\right)\right|<\frac{\varepsilon}{2} .
$$

The final two inequalities imply

$$
1 \int_{\Omega}\left|\varphi\left(\tau w_{1}+u_{n}\right)\right| \leqq \int_{\Omega \cap\left\{\left|u_{n}\right|<k\right\}}\left|\varphi\left(\tau w_{1}+u_{n}\right)\right|+\frac{1}{k} \int_{\Omega \cap\left\{\left|u_{n}\right|>k\right\}}\left|u_{n} \varphi\left(\tau w_{1}+-u_{n}\right)\right|<\varepsilon .
$$


Using Vitali's theorem we have

$$
\varphi\left(\tau w_{1}+v(\tau)\right) \in L^{1}\left(\mathbf{I}^{2}\right) \text { and } \varphi\left(\tau w_{1}+u_{n}\right) \stackrel{L^{1}\left(\mathbf{I}^{2}\right)}{\longrightarrow} \varphi\left(\tau w_{1}+v(\tau)\right) .
$$

This means that $(\tau, v(\tau))$ fulfill (4.4) and the proof of Lemma 4.2 is completed (essentially the same procedure can be found in STrauss [9])

We go on proving Theorem 4.1. Put

$$
\begin{aligned}
& \mathbf{S}=\left\{(\tau, \dot{v}(\tau)) \in \mathbf{R} \times \mathscr{H}_{0}:(\tau, \dot{v}(\tau)) \text { fulfill }(4.4)\right\}, \\
& \mathbf{S}_{n}=\left\{\left(\tau, u_{n}\right) \in \mathbf{R} \times \mathbf{V}_{n}: u_{n} \text { is the Galerkin solution of }(4.4)\right\} .
\end{aligned}
$$

It is easy to see that the GPSs of (4.1) are such $u=\tau w_{1}+v(\tau)$ that $(\tau, v(\tau)) \in \mathrm{S}$ and

$$
-\lambda \tau+\int_{\mathbf{I}^{2}} \varphi\left(\tau w_{1}+v(\tau .)\right) w_{1}=s
$$

Let us define a continuous function (see $[3,4,8]$ )

$$
\dot{F}: \mathscr{P}=\mathbf{S} \cup\left(\bigcup_{n=1}^{\infty} S_{n}\right) \rightarrow \mathbf{R}
$$

by the relation.

$$
F(\dot{\tau}, v)=-\dot{\lambda} \tau+\int_{\mathbf{I}} \varphi\left(\tau w_{1}+v\right) w_{1} \cdot
$$

Since $\varphi(z) \geqq \dot{0}(z \in \mathbf{R})$ and $w_{1}>0$ on $\mathbf{l}^{2}$, we obtain

$$
F(\tau, v) \geqq-\dot{\lambda} \tau,
$$

for all $(\tau, v) \in \mathscr{S}$. Using (4.8) we obtain

$$
F(\tau, v) \geqq-\lambda \tau+\int_{\mathbf{1}} \frac{\lambda}{\alpha^{2}}\left(\tau w_{1}+v\right) w_{1}-\gamma \int_{\mathbf{1}} w_{1}=\left(\frac{\lambda}{\alpha^{2}}-\lambda\right) \tau-\gamma \int_{\mathbf{I}^{2}} w_{1},
$$

for all $(\tau, v) \in \mathscr{S}$. Since $0<\alpha,<1,(4.11)$ and (4.12) imply

$$
\lim _{\tau \rightarrow \pm \infty} F(\tau, v)=+\infty
$$

uniformly with respect to such $v \in \mathscr{H}_{0}$ that $(\tau, v) \in \mathscr{P}$.

Put

$$
T_{2}\left(h_{1}\right)=\sup _{\substack{(\tau, v) \in \cup S_{n} \\ r \in[-1.1]}} F(\tau, v)
$$

From Lemma 4.1 we obtain $T_{2}\left(h_{1}\right)<+\infty$. Suppose $s>T_{2}\left(h_{1}\right)$. By (4.13) there exists $\tau_{0} \in \mathbf{R}$ such that

$$
\inf _{\substack{(\tau, v) \in \cup \mathbf{S}_{n} \\ \tau \in\left(-\infty,-\tau_{0}\right] \cup\left[\tau_{0},+\infty\right)}} F(\tau, v)>s .
$$

Using the assertion of Leimma 3.1 (see also $[3,4]$ ), we obtain for each $n \in \mathbf{N}$ the existence of a connected subset $\tilde{\mathbf{S}}_{n} \subset \mathrm{S}_{n}$ such that $\left[-\tau_{0}, \tau_{0}\right] \subset \operatorname{proj}_{\mathbf{R}} \widetilde{\mathrm{S}}_{n}$. Then according to the definition of $T_{2}\left(h_{1}\right)$ by (4.14), for each $n \in N$ there exists $\tau_{n}{ }^{1} \in(-\infty,-1)$, $\tau_{n}{ }^{2} \in(1,+\infty)$ and $v_{n}{ }^{1}, v_{n}{ }^{2} \in \mathscr{H}_{0}$ such that

$$
\left(\tau_{n}^{i} ; v_{n}^{i}\right) \in S_{n}(i=1,2) \text { and } F\left(\tau_{n}^{i}, v_{n}^{i}\right)^{j}=s \text {. }
$$


By (4.13), $\left\{\tau_{n}^{1}\right\}_{n-1}^{\infty} \subset(-\infty,-1),\left\{\dot{\tau}_{n}^{2}\right\}_{n-1}^{\infty} \subset(1,+\infty)$ are bounded sequences and Lemma 4.1 implies that $\left\{v_{n}^{i}\right\}_{n-1}^{\infty} \subset \mathscr{H}_{0}(i=1 ; 2)$ are also bounded. After possibly passing to a suitable subsequence, we may suppose (by the same argument as in the proof of Lemma 4.2) that

$$
\tau_{n}^{i} \rightarrow \tau^{i}, \quad v_{n}^{i} \rightarrow v^{i}
$$

and by the same procedure as in the proof of Lemma 4.2 we prove that $\left(\tau^{i}, v^{i}\right) \in \mathrm{S}$ $(i=1,2)$. Since $\tau^{1} \neq \tau^{2}$ and $F^{\prime}\left(\tau^{i}, v^{i}\right)=s(i=1,2)$, the functions $u_{i}=\tau^{i} w_{1}+v^{i}$ are two distinct GPSs of (4.1). This fact proves the assertion (i), of 'Theorem 4.1.

Put

$$
T_{1}\left(h_{1}\right)=\inf _{(\tau, v) \in \mathrm{S}} F(\tau, v)
$$

From (4.11), (4.12) we obtain $T_{1}\left(h_{1}\right)>-\infty$. If $s<T_{1}\left(h_{1}\right)$ then (4.1) has no GPS, which proves the assertion (iii).

By the assertion (i) there is a sequence $\left\{s_{m}\right\}_{m-1}^{\infty} \subset\left(T_{2}\left(h_{1}\right),+\infty\right), s_{m} \rightarrow T_{2}\left(h_{1}\right)$ such that there exist bounded sequences $\left\{\tau_{m}\right\}_{m=1}^{\infty} \subset \mathbf{R},\left\{v_{m}\right\}_{m=1}^{\infty} \subset \mathscr{H}_{0}$ such that $\left(\tau_{m}, v_{m}\right) \in \dot{\mathbf{V}}_{m}$. .

- $F\left(\tau_{m}, v_{m}\right)=s_{m}$. After possibly passing to a subsequence, we can suppose that

$$
\tau_{m} \rightarrow \tau_{0}, \quad v_{m} \stackrel{H}{\rightarrow} v_{0} \in \mathscr{H}_{0} .
$$

By the same procedure as in the proof of Lemma 4.2 we prove that $\left(\tau_{0}, v_{0}\right) \in \mathrm{S}, F\left(\tau_{0}, v_{0}\right)$ $=T_{2}\left(h_{1}\right)$. Then $T_{2}\left(h_{1}\right) \in \mathbf{M}\left(h_{1}\right)$, which proves the assertion (ii). The proof of Theorem 4.1 is completed

Remark 4.1: There are no restrictions to the growth of $\varphi$ in $+\infty$ and so the Nèmytskij's operator

$$
u \mapsto \varphi(u)
$$

is not always acting from $\mathbf{H}$ into $\mathbf{H}$. This is the reason why we use the Galerkin method and the properties of Brouwer degree (instead of the Leray-Schauder degree) to prove the existence and multiplicity of solutions.

\section{REFERENCES}

-[1] Amann, H., Ambrosetri, A., and G. Maxcini: Elliptic equations with noninvertible Fredbolm linear part and bounded nonlinearities. Math. Z. 158 (1978), 179-194.

[2] Drábek, P.: Bounded nonlinear perturbations of second order linear elliptic problems. Comment. Math. Univ. Carolinac 22, 2 (1981), 215-221.'

- [3] Drábek, P.: Solvability of the superlinéar elliptic boundary value problem. Comment. Math. Univ. Carolinae 22, 1 (1981), 27-35.

[4] Dráber, P.: Nonlinear elliptic problems with jumping nonlinearities near the first eigenvalue. Aplikace matematiky 26 (1981), 304-311.

[5] Fradeiredo, D. G., and Wei Ming Ni: Perturbations of second order linear elliptic problems by nonlinearities without Landesman-Lazer condition. Nonlinear Analysis and Appl. 3 (1979), 629-634.

[6] Fuč́n, S.: Nonlinear noncoercive problems. In: Conferenze del Seminario di Matematica Dell'Universitá di Bari, No. 166, Bari 1979, p. $301-353$.

[7] Fučrk, S., Neč́as, J., and. V. SovǒEK: Einführung in dic Variationsrechnung. Teubner Texte für Mathematik. Teubner: Leipzig 1977. 
[8] Hess, P., and B. RUF: On a superlinear elliptic boündary value problem. Math. Z. 164 (1978), 9-14.

[9] Stradss, W.: On weak solutions of semilinear hyperbolic equations. An Acad. Brasil. Ci. $42(1970), 645-651$.

[10] Vejvoda, O. et al.: Partial differential equations: time-periodic solutions. Sijthoff Nordhoff, The Netherlands_1981.

Manuskripteingang: 20.02.1981; Eingang der revidierten Fassung: 3. 11. 1981

\section{VERFASSER:}

Dr. Pavel Dráber

Katedra matematiky VSSE

ČSSR - 30614 Plzeň, Nejedlého sady 14 